\title{
Role of immunotherapy in stage IIIA non-small cell lung cancer: a narrative review
}

\author{
Virginia Calvo $^{1}$, Belén Sierra-Rodero ${ }^{2,3}$, Alberto Cruz-Bermúdez ${ }^{2}$, Mariano Provencio ${ }^{1}$ \\ ${ }^{1}$ Department of Medical Oncology, Hospital Universitario Puerta de Hierro-Majadahonda, Madrid, Spain; ${ }^{2}$ Instituto de Investigación Sanitaria \\ Puerta de Hierro-IDIPHISA, Majadahonda, Madrid, Spain; ${ }^{3} \mathrm{PhD}$ Programme in Molecular Biosciences, Doctoral School, Universidad Autónoma de \\ Madrid, Madrid, Spain \\ Contributions: (I) Conception and design: All authors; (II) Administrative support: None; (III) Provision of study materials or patients: None; (IV) \\ Collection and assembly of data: None; (V) Data analysis and interpretation: None; (VI) Manuscript writing: All authors; (VII) Final approval of \\ manuscript: All authors. \\ Correspondence to: Mariano Provencio. Department of Medical Oncology, Puerta de Hierro Hospital, Manuel de Falla Street \#1, 28222 Majadahonda, \\ Madrid, Spain. Email: mprovenciop@gmail.com.
}

\begin{abstract}
Non-small cell lung cancer (NSCLC) is one of the main leading causes of death by cancer worldwide. Approximately one third of cases are diagnosed at stage III, whose therapeutic strategy based on multimodal treatment has reached a plateau of effectiveness. Treatment with anti-PD-1/PD-L1 [antiPD-(L)1] antibodies have been stablished in advanced stages due to their improved clinical benefit and acceptable profile of related adverse events. Lately, numerous clinical trials have been designed with diverse approaches to introduce immunotherapy into stages IIIA. The objective of this review is to discuss the different strategies that are being taken in the ongoing clinical trials, taking into account the peculiarities of stage IIIA and the tumor microenvironment. PubMed, major international conferences and clinicaltrials. gov databases were searched from January 1, 1990, to May 11, 2020 using the terms NSCLC, stage III, resectable, unresectable, immunotherapy, checkpoint inhibition, neoadjuvant and adjuvant. In this review we describe, in the context of stage IIIA disease, the impact of anti-PD-(L)1 and conventional therapies on the tumor microenvironment. Moreover, we describe the role of immunotherapy, through the heterogeneous landscape of stage IIIA NSCLC, summarizing ongoing clinical trials in incidental, potentially resectable, and unresectable tumors. We finally discuss the potential of PD-L1, tumor mutation burden (TMB), and T-cell receptor (TCR) repertoire analysis as predictive biomarkers in stage IIIA NSCLC. Initial results seem to favor neoadjuvant over adjuvant treatment, with chemoimmunotherapy combination standing out. Ongoing trials will answer whether immunotherapy can transform stage IIIA from lethal to a curable disease. Additionally, higher pathological response rates observed, open the possibility to establishing it as survival surrogate, speeding up the potential immunotherapy implementation in stage IIIA NSCLC.
\end{abstract}

Keywords: Lung cancer; immune checkpoint inhibitors; immunotherapy; locally advanced; stage IIIA

Received: 15 April 2020; Accepted: 08 July 2020; Published: 25 November 2021.

doi: $10.21037 /$ ccts-20-82

View this article at: http://dx.doi.org/10.21037/ccts-20-82

\section{Introduction}

Lung cancer is the leading cause of cancer-related death in both genders worldwide (1). Approximately $85 \%$ of lung cancer cases are non-small cell lung cancer (NSCLC). Of patients with NSCLC, at diagnosis, $20 \%$ present with stage I or II, whereas $30 \%$ present with stage III, locally advanced disease, and $50 \%$ of patients with stage IV disease. Five-year survival rate of patients with stage I NSCLC is approximately $70-90 \%$, whereas stage II to III NSCLC patients, have a 5 -year survival rate of approximately $25 \%$ to $60 \%$ (2).

Stage III NSCLC is a heterogeneous disease. In the 
$8^{\text {th }}$ edition of the TNM classification, stage III NSCLC includes $\mathrm{M} 0$ patients, who present $\mathrm{N} 2$ or $\mathrm{N} 3$ disease, a tumor with $\mathrm{T} 4$ features or one categorized as $\mathrm{T} 3 \mathrm{~N} 1$ (3). Stage IIIA includes T4N0 and T3/4N1 tumors as well as T1/T2 N2 tumors.

Thus, the stage IIIA management is complex including patients with resected, potentially resectable and unresectable tumors and therefore their treatment should be deliberated by a multidisciplinary team (4). Outcomes remain poor, even in the case of potentially resectable tumors, with a median progression-free survival (PFS) of 13 months and a 3 -year overall survival (OS) rate of $30 \%$ for this subset of patients, without major changes in the last 25 years (5).

In the last few years, cancer immunology knowledge has experienced a remarkable advance, leading to use immunotherapy against cancer (6). In March 2015, the FDA approved an anti-PD-1 antibody (Nivolumab) as a second-line treatment in metastatic NSCLC since it produces a significant increase in overall survival (OS) of stage IV patients. Since then, this type of treatment has been positioned as the first choice for different histologies, becoming the main therapy in advanced stages of NSCLC (7). At the present time, immunotherapy has new challenges ahead, as the stage IIIA scenario.

There are currently dozens of phase two and three clinical trials, both monotherapy and immunotherapy combinations, addressing this complex task of bringing immunotherapy closer to stage IIIA clinical practice, the results of which will be known during the next few years.

Therefore, in this changing scenario of great development and clinical relevance, it is necessary to discuss the current knowledge and the possible role of immunotherapy in stage IIIA.

For this purpose, in this narrative review we describe the tumor microenvironment as therapy target, as well as, the different strategies that are being taken in the ongoing clinical trials, taking into account the specific vicissitudes of stage IIIA NSCLC. We present the following article in accordance with the Narrative Review reporting checklist (available at https://ccts.amegroups.com/article/ view/10.21037/ccts-20-82/rc).

\section{Methods}

We searched PubMed from January 1, 1990, to May 11, 2020, employing the following search words alone or in combination: NSCLC, stage III, resectable, unresectable, immunotherapy, checkpoint inhibition, neoadjuvant and adjuvant.

Additionally, relevant ongoing clinical trials included in this review were found in clinicaltrials.gov database, using "stage III NSCLC" as search term, and limiting the search to completed, not yet recruiting, recruiting, enrolling by invitation, and active but not recruiting enrolling status. Further information was collected by consulting major international conferences (IASLC, ESMO and ASCO meeting databases), applying the name of the trial and NTC number.

\section{Discussion}

\section{Treatments and tumor immune microenvironment}

An emerging hallmark of cancer is the cancer cell's ability to avoid destruction by the immune system, known as Immunoevasion. The three general categories of immunoreactive mechanisms include: an insufficient number of T-cells generated within the lymphoid compartment; an insufficient number of T-cells extravasating into the tumor; and inhibition of T-cells in the tumor microenvironment. The tumor microenvironment, in turn, offers three main immunoreactive tools: surface membrane proteins that function as immune checkpoints, including PD-1, CTLA4, lymphocyte-activation gene 3 (LAG-3) protein, T-cell immunoglobulin and mucin domain-containing protein 3 (TIM-3), B- and T-lymphocyte attenuator (BTLA), and the adenosine $\mathrm{A} 2 \mathrm{a}$ receptor $(\mathrm{A} 2 \mathrm{aR})$; the relationship between selected soluble factors and metabolic alterations, such as IL10 , transforming growth factor beta, adenosine, indoleamine 2,3-dioxygenase (IDO), and arginase; and inhibitory cells, including cancer-associated fibroblasts $(\mathrm{CAFs})$, regulatory T-cells, myeloid-derived suppressor cells (MDSCs), and tumor-associated macrophages (TAMs) (8-11).

\section{Anti-PD-1/PD-L1 and tumor immune}

\section{microenvironment}

PD-L1 overexpression has been observed in $30-50 \%$ of all NSCLC tumors across all stages and histologies. However, PD-L1 positivity has been associated with male gender, smoking status, higher $\mathrm{T}$ and $\mathrm{N}$ status, advanced tumor grade and stage, and wild-type epidermal growth factor receptor (EGFR) $(12,13)$.

Anti-PD-1/PD-L1 [anti-PD-(L)1] antibodies activity is based on the blockade of $\mathrm{PD}-1$ protein in lymphocytes or PD-L1 in tumor cells, preventing lymphocytes 
inactivation and promoting tumor elimination. A great part of the knowledge of its mechanism is due to neoadjuvant immunotherapy studies. In the situation with the intact tumor, on one side, anti-PD-(L)1 rejuvenates the tumor-specific cytotoxic T-cells from the tumor microenvironment, causing them to activate, proliferate and mobilize to eliminate distant micrometastasis. Additionally, anti-PD-(L)1 increase tumor antigen presentation by dendritic cells in the tumor draining lymph nodes activating new tumor-specific T-cells that then migrate to tumor sites (14). Both processes trigger a powerful systemic antitumor immune response and the generation of memory T-cells that may provide long-term protection (15-18). Conversely, the neoantigen repertoire is reduced when the primary tumor is resected, limiting this anti-tumor immune response in the adjuvant setting and representing a strong argument for neoadjuvant approach. Moreover, several immunological pathways are disrupted by surgical stress (19). While essential for wound healing, surgical stress leads to expansion of regulatory T-cells, MDSC, and M2 macrophages, resulting in an overall state of immunosuppression with PD-1/CTLA-4 increase and T-cell exhaustion. Immune checkpoint inhibitors (ICI) in neoadjuvant setting might be advantageous activating tumor infiltrating T-cells prior to surgery, and avoiding PD-1 expression on immune cells in the postoperative period.

However, the impact of anti-PD-(L) 1 antibodies is true as long as there are no other elements governing the activity of cytotoxic lymphocytes as mentioned before (20). Perhaps for this reason, the presence of PD-L1 in tumor cells has not been a perfect marker of response to treatment, so that today, it is not clear which patients will benefit more from the treatment (21).

\section{Chemo-radiotherapy and tumor immune microenvironment}

Although cancer chemo-radiotherapy has generally been associated to immunosuppression, it is now established that certain drugs, such as paclitaxel, cisplatin, carboplatin and gemcitabine, as well as, specific tumor radiation, can regulate and modulate antitumor immunity. In fact, many studies are being based on chemo-radioimmunotherapy combinations (22-24). Chemo-radiotherapy has the ability to obtain an anti-tumor immune response by inducing immunogenic tumor cell death and subsequent release of tumor-associated antigens, which ultimately activates antigen-presenting cells (25).

NSCLC tumors after neoadjuvant chemotherapy presented higher levels of PD-L1 ${ }^{+}$tumor cells and tumor infiltrating lymphocytes than those who underwent upfront surgery without neoadjuvant treatment. Higher levels of helper T-cells and TAMs were associated to increased survival in NSCLC patients treated with neoadjuvant chemotherapy (26).

Paclitaxel increase the levels of $\mathrm{CD}^{+}{ }^{+} \mathrm{T}$-cells secreting IFN $\gamma$ as well as $\mathrm{CD}^{+}{ }^{+}$T-cells secreting IL-2, associated to antitumor immune responses (27). Additionally, paclitaxel hampers regulatory T-cells viability and immunosuppressive cytokine production maintaining $\mathrm{CD}^{+}$effector T-cell function (28).

Platinum chemotherapy alters the levels of myeloid cells increasing dendritic cells and reducing MDSCs, thus favoring immune effector responses. Decreased iNOS, IDO and IL4R expression after platinum-based therapy has been observed in a serial analysis of blood samples from NSCLC patients (29).

Ablative radiation therapy increases immunogenic tumor cell death and T-cell priming in draining lymphoid tissues promoting tumor elimination. As a consequence, distant metastases may be eliminated in a $\mathrm{CD} 8^{+} \mathrm{T}$-cell dependent fashion, a process denominated abscopal effect, which is not well understood yet (22).

\section{Incidental stage IIIA}

Incidental stage IIIA includes patients with previously unknown N2 disease revealed during surgery. The reduced local control and OS after surgery in these patients makes them suitable for adjuvant chemotherapy. Adjuvant platinum-based chemotherapy has been associated to a $5 \%$ OS increase at 5 years $(30,31)$.

The impact of radiotherapy after surgery is still under debate since it diminish local relapse risk without improving OS $(32,33)$. Currently, for stage II-IIIA resectable NSCLC the standard treatment is still radical surgery (lobectomy/ pneumonectomy) followed by 4 cycles of adjuvant chemotherapy (CT) with a platinum-based doublet (34). Despite the available treatments, the survival of completely resected NSCLC remains poor and this is the reason why it is necessary to evaluate new strategies of management.

The use of ICI in the adjuvant setting is evaluated in different solid tumors such as lung, bladder, esophageal, colorectal, ovarian cancer and some others (35). However, melanoma is the first tumor in which immunotherapy has been demonstrated efficacy after surgery. The first ICI approved in this context was ipilimumab (anti-CTLA4) (36), 


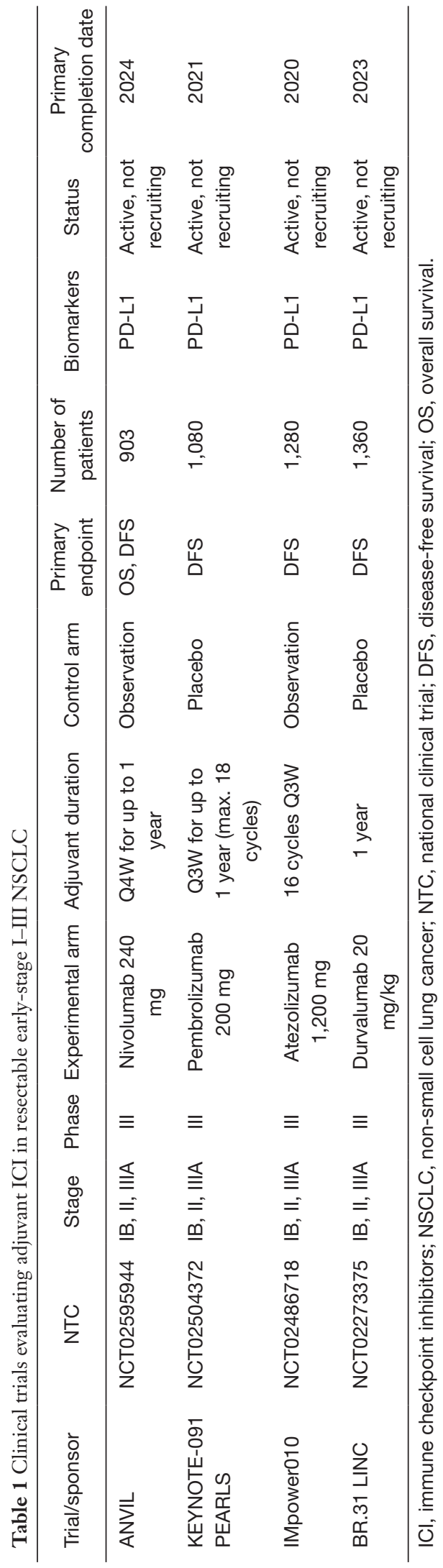

followed by nivolumab (37) and pembrolizumab (antiPD-1) (38).

Currently, four randomized phase III clinical trials which are evaluating the role of immunotherapy in patients with completely resected NSCLC stand out. Two of them use antibodies anti PD-1 (ANVIL and PEARLS) and two more with anti PD-L1 (IMpower-010 and NCT02273375) (Table 1). These trials enroll early stage (IB $>4 \mathrm{~cm} / \mathrm{II} / \mathrm{IIIA}$ ), complete resected NSCLC patients regardless of tumor PD-L1 level. The adjuvant ICI therapy is administered up to one year and the common primary end point is diseasefree survival (DFS).

ANVIL trial (NCT02595944) (39), is a phase III, randomized trial comparing adjuvant nivolumab with observation after surgical resection and standard of care adjuvant chemotherapy and/or radiotherapy for patients with resected stage IB-IIIA NSCLC. The primary endpoints DFS and OS are currently being evaluated.

Keynote-091 (NCT02504372) (40), is a phase III, randomized trial comparing pembrolizumab versus placebo, after standard of care adjuvant chemotherapy in patients with resectable, early-stage NSCLC. The primary endpoint is DFS.

IMpower010 (NCT02486718) (41), is a phase III, randomized, open-label trial recruiting stage IB-IIIA NSCLC resected patients. The trial will evaluate the safety and efficacy of atezolizumab versus best supportive care following adjuvant cisplatin-based chemotherapy. The primary endpoint is DFS, and secondary endpoints include OS and safety.

BR.31. LINC (NCT02273375) is a phase III, randomized trial comparing adjuvant durvalumab administration versus placebo after completely resected NSCLC. The clinical trial is evaluating DFS as primary endpoint.

The possible limitations of these studies include: difficult recruitment (especially the placebo-controlled studies when patients realize they may be coming to the hospital for one-year placebo administration); difficult treatment compliance; unknown duration of treatment; lack of predictive biomarkers; DFS as primary endpoint instead of OS and furthermore, as in other adjuvant studies, inability to define short term surrogate outcomes such as pathologic responses, which implies years to reach conclusions.

\section{Potentially resectable stage IIIA}

In the chemotherapy era, the neoadjuvant treatment has theoretical advantages like: assess of response to 
chemotherapy in vivo and this in turn helps identify patients who will potentially benefit from this therapy; perhaps the better locoregional drug delivery because of intact vessels presurgery; better tolerability; early treatment of micrometastatic disease; downstaging with improved resectability and offers an excellent framework for clinical and molecular surrogate markers discovery. However neoadjuvant therapy has potential disadvantages: delay in local therapy due to toxicity, risk progression in chemoresistant patients and pre-operative complications.

Neoadjuvant chemotherapy has not been evaluated as extensively as postoperative. However, several phase III studies have shown that platinum-based induction chemotherapy increases OS (42-44). In stage IIIA (N2) patients, induction chemotherapy increases OS compared to surgery alone $(45,46)$. These results have been confirmed in a later meta-analysis (47). A meta-analysis with 15 randomized trials showed a significant benefit of preoperative chemotherapy on OS [HR 0.87 (0.78-0.96), $\mathrm{P}=0.007]$, demonstrating a 5 -year OS rate increase of $5 \%$. Neoadjuvant chemotherapy has comparable influence on OS than adjuvant chemotherapy, however more available results support the use of adjuvant treatment.

\section{Surrogate markers of efficacy outcome}

The use of OS as primary endpoint in resectable NSCLC clinical trials has occasioned prolonged duration of these trials, with the consequent high cost associated and slow development of new therapies. Regardless of their neoadjuvant or adjuvant approach, time from enrollment to publication was in the range of 9 to 13 years. Thus, for faster development of new therapies in early stages of NSCLC there is a need for surrogate markers that anticipate DFS and OS historical endpoints. The assessment of these surrogate markers of efficacy outcome, whether these are clinical, pathological or based in biological parameters, is best analyzed in the neoadjuvant scenario.

Complete surgical resection $(48,49)$, tumor downstaging (50) and complete and major pathologic responses (CPR and MPR, respectively) $(51,52)$ after neoadjuvant chemotherapy, have been associated with improved survival in resectable NSCLC. MPR was first described in the chemotherapy era by at Junker et al. Patients with less than $10 \%$ viable tumor cells after neoadjuvant treatment (regression grades $\geq \mathrm{IIb}$ ) had higher 3-year OS rates than those with more than $10 \%$ of viable tumor cells in the resection specimen $(52 \%$ vs. 9\%, $\mathrm{P}=0.02$ ) (53). The robust survival improvement in patients who had MPR compared to other groups was shown by Pataer et al. in a broad analysis of 192 stage I-III NSCLC patients resected after neoadjuvant chemotherapy. This study demonstrated that each additional percentage of remaining tumor cell after treatment was linked to a $1 \%$ increase in both, the risk of death $(\mathrm{HR}=1.01, \mathrm{P}=0.005)$ and the risk of disease progression ( $\mathrm{HR}=1.01, \mathrm{P}=0.01$ ) (52). Although MPR after induction chemotherapy is significantly associated with hazard ratio for death, its application has not been validated in NSCLC probably due to the low rates of major or complete pathological response achieved with induction chemotherapy, generally close to $20 \%$ for major responses and $4 \%$ for complete responses (ranging from $0 \%$ to $16 \%)(31,42,47,51,54)$. This limitation derived from its low frequency could be solved in the near future due to the observed increase of major and complete pathological responses in patients receiving neoadjuvant immunotherapy $(17,23)$. Additionally, pathologic response criteria adapted to ICI are being evaluated $(55,56)$, as well as clinical responses (57), since strong discrepancies between pathological and clinical responses are often observed $(17,58)$. Future studies will shed light in whether major or complete pathologic responses are correlated to survival also in the immunotherapy context and could be implemented as PFS and OS surrogate. This may allow for a faster readout of long-term benefits than in adjuvant studies.

\section{Clinical trials}

Multiple ICI have been evaluated as neoadjuvant treatment, but their use in this setting remains investigational (Table 2).

Recently, Forde, et al. (NCT02259621) assessed the feasibility of neoadjuvant PD-1 blockade in a study with 21 NSCLC patients (stage I to IIIA) (17). Two doses of the anti-PD-1 inhibitor nivolumab $(3 \mathrm{mg} / \mathrm{kg})$ were administered intravenously every two weeks. Tumor resection was scheduled 4 weeks after neoadjuvant initiation. The study showed no ICI delivery complications with no surgery delay and no relevant postoperative complications. Treatment-related adverse events (TRAEs) of any grade were described in $23 \%$ of patients with only one TRAE of grade $\geq 3$. MPR was determined in $45 \%$ of patients who underwent surgery and $13 \%$ of patients had CPR. However, only $10 \%$ of patients had objective clinical responses on post-treatment computer tomography scans (CT-scans). MPR occurred regardless of PD-L1 positivity and tumor mutational burden (TMB) was predictive of pathologic response. Using multiplex immunofluorescence staining a large inflammatory component was observed composed of $\mathrm{CD} 8^{+}$T-cells, PD- $1^{+}$cells. At the same time, the study of 
the peripheral blood showed a systemic immune response in these patients, composed of T-cells with T-cell receptors (TCRs) common to those found in the tissue. At median follow up of 30 months, 5 patients had disease progression and 2 patients have died. The 24 months recurrence-free survival rate is $69 \%$ (95\% CI, 51 to 93 ) (59).

LCMC3 trial (NCT02927301) $(60,61)$, is a phase II single-arm study of neoadjuvant anti-PD-L1 (atezolizumab) in resectable (stages IB to selected IIIB, T3N2) NSCLC patients. For the last interim efficacy analysis (5 Sep 2018 data cut) they reported on the first 101 of 180 planned patients (61). By RECIST, 6/82 patients had partial response, 72 had stable disease and 4 had progressive disease. The MPR rate was $18 \%$ (95\% CI, 11 to 28 ) 15/82, 4 patients had CPR (5\%). There was one unrelated grade 5 adverse events (AEs) and 16 grade 3 or 4 AEs (three treatment related), and surgery was delayed in one patient due to grade 3 immune-mediated pneumonitis. The biological correlative studies are ongoing.

NEOSTAR study (NCT03158129) is a phase II study of ICI induction for untreated stage I-IIIA (single N2) NSCLC patients. Three doses of preoperative nivolumab $3 \mathrm{mg} / \mathrm{kg}$ monotherapy or in combination with ipilimumab $1 \mathrm{mg} / \mathrm{kg}$ are administered to patients every two weeks (62). Five of the 31 patients did not undergo tumor resection due to different causes (one had hypoxemia grade 3, two had high surgical risk and two were no longer resectable). MPR rate in the remaining patients was $28 \%$ and $31 \%$ in the nivolumab or nivolumab plus ipilimumab trial arms, respectively. In ASCO 2019 updated data were presented (63) showing 39 of 44 underwent surgery with $89 \%$ resectability. The MPR rate was $24 \%$ overall, $17 \%$ with nivolumab and $33 \%$ with the combination therapy. Secondary AEs were 4\%, including 2 bronchopleural fistulas and 8 air leaks.

Chemoimmunotherapy has demonstrated superiority compared with chemotherapy alone in patients with metastatic NSCLC. This led to study the role of chemoimmunotherapy with surgery in earlier-stage NSCLC.

NADIM trial (NCT03081689) (23) is a prospective, open-label, single-arm phase II trial, evaluating the safety and efficacy of neoadjuvant chemotherapy (paclitaxel $200 \mathrm{mg} / \mathrm{m}^{2}+$ carboplatin AUC 6 IV every 3 weeks) plus nivolumab $(360 \mathrm{mg}$ ) followed by adjuvant nivolumab (240 mg IV every 2 weeks for 4 months and $480 \mathrm{mg}$ IV every 4 weeks for 8 months) in 46 patients with resectable stage IIIA (N2 or T4) NSCLC. The primary endpoint was PFS at 24 months. Efficacy was evaluated using objective pathological response criteria. The last results were presented in WCLC 2019 (58). Forty-one of 46 patients had undergone surgery and all tumors were resectable with $\mathrm{R} 0$ resection. Intention to treat analysis showed that 34 patients $(83 \%, 95 \%$ CI, 68 to 93$)$ achieved MPR of which 24 (59\%, 95\% CI, 42 to 74) were CPR. This CPR rate is the highest ever seen in this context. Downstaging was observed in 38 (93\%, $95 \%$ CI, 80 to 98 ) of cases. The median follow-up was 13.8 and 12 months PFS was $95.7 \%$ (95\% CI, 84 to 99). This is the first multi-center study to explore chemotherapy and immunotherapy in the neoadjuvant setting in stage IIIA. A new randomized phase II clinical trial comparing the same neoadjuvant chemotherapy plus nivolumab schema followed by a shorter adjuvant nivolumab monotherapy for 6 months, $v s$. standard chemotherapy alone, is currently ongoing (NADIM II, ClinicalTrials.gov number, NCT03838159).

The study of Shu and collaborators, NCT02716038, is another study with combined chemotherapy and ICI therapy. In this trial, four cycles of atezolizumab plus carboplatin-nabpaclitaxel reported an MPR in 17 (57\%) of 30 patients included. The most common grade 3-4 AEs were neutropenia, thrombopenia and transaminases elevation (64).

This led to several ongoing phases III trials with this approach (Table 2). They all differ in certain aspects of their design.

The KEYNOTE-671 trial (NCT03425643) (65), double-blind, randomized 1:1 placebo-controlled, phase III trial testing four cycles of concomitant neoadjuvant platinum doublet plus pembrolizumab followed by surgery and 13 cycles of adjuvant pembrolizumab for resectable stage IIB or IIIA NSCLC patients. Although its solid design, using event-free survival and OS as primary endpoints, the adjuvant placebo administration can be a drawback for patient recruitment.

The open-label Checkmate-816 trial (NCT02998528) (66), evaluates the safety and effectiveness of neoadjuvant nivolumab plus platinum-doublet followed by surgery and postoperative standard of care $v s$. chemotherapy alone. Initially, the study planned an additional arm testing nivolumab plus ipilimumab combination, however it was closed on December 2018. This study will compare EFS and CPR rate among participants treated with neoadjuvant nivolumab plus platinum doublet chemotherapy $v s$. participants treated with platinum doublet chemotherapy in stage IB-IIIA NSCLC.

The IMpower-030 (NCT03456063) (67) is a double- 


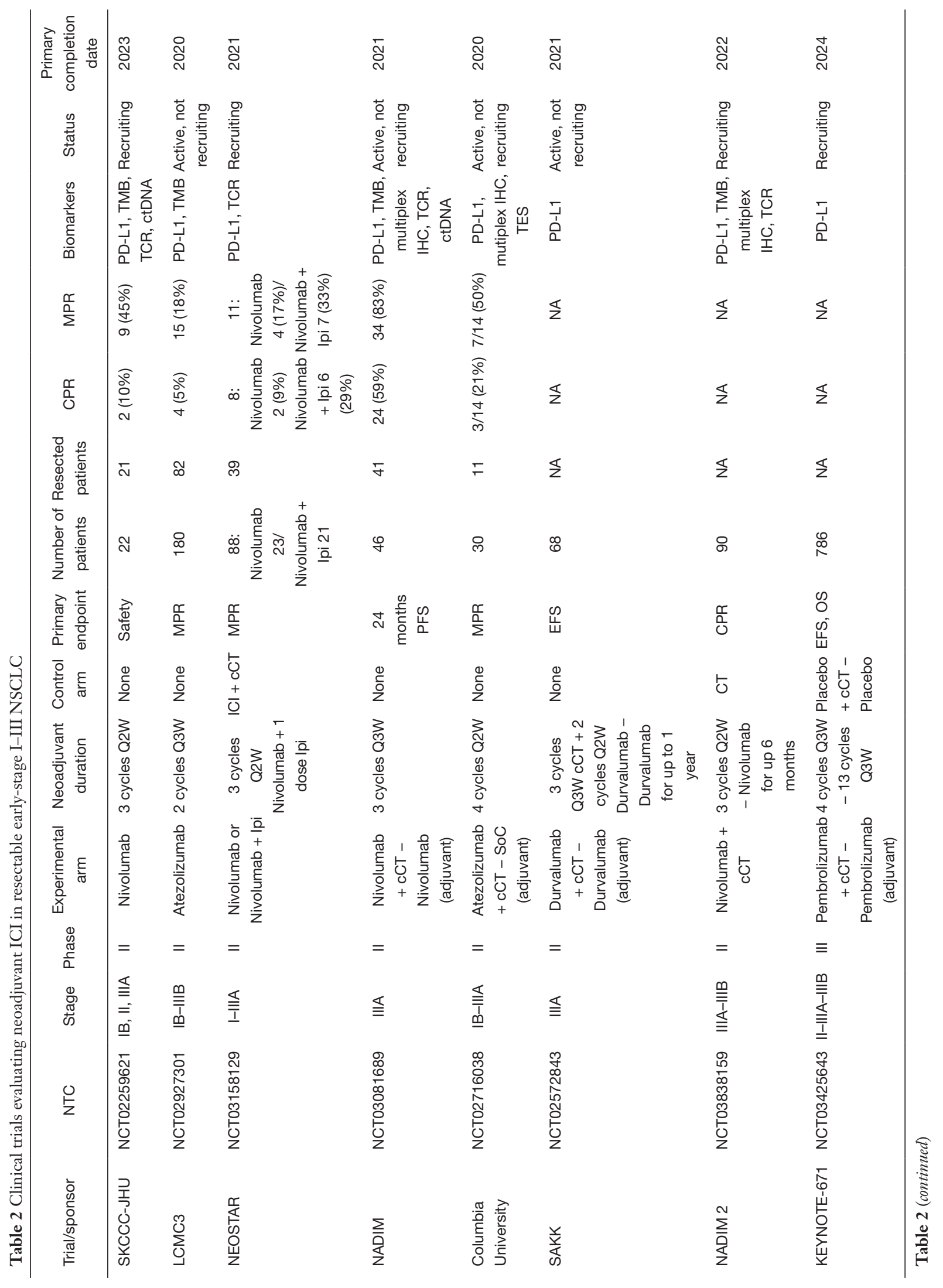




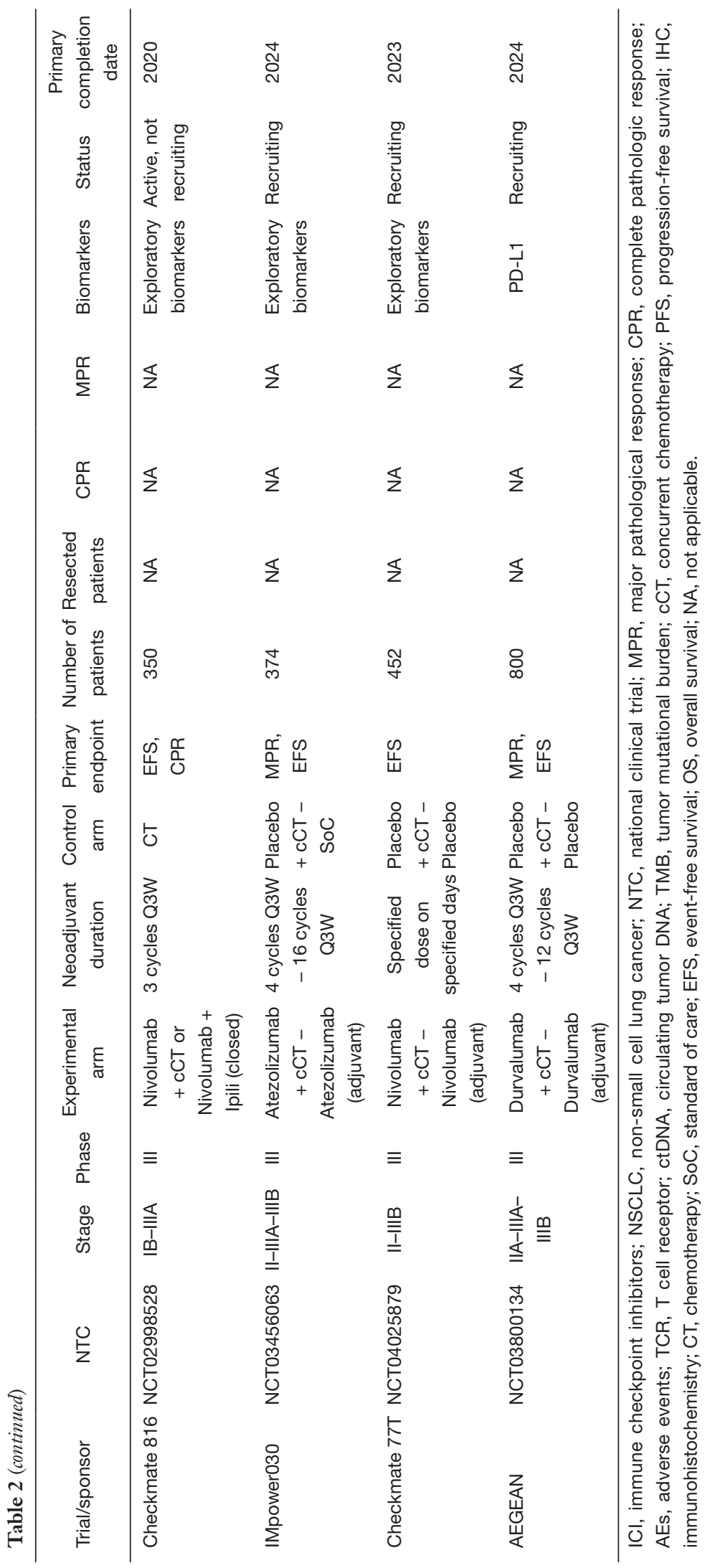


blind, randomized study of resectable stage II, IIIA and IIIB (T3N2) NSCLC patients. The study will determine the efficacy of 4 cycles of neoadjuvant atezolizumab $(1,200 \mathrm{mg}$ Q3W), in combination with a platinum-based doublet followed by surgery and postoperative atezolizumab $v$ s. neoadjuvant platinum-based doublet followed by surgery and supportive care. Trial has MPR as primary endpoint.

\section{Unresectable tumors}

Most patients diagnosed with stage III NSCLC are considered inoperable by a multidisciplinary team for both medical and anatomical reasons. Local-regionally unresectable NSCLC tumors have been traditionally treated with concurrent chemoradiation, obtaining more clinical benefit than sequential therapy or radiation alone (68-70). Consequently, weekly low-dose platinum-taxane or platinum doublet used as concurrent chemoradiation has been the standard of care until very recently, but only $15-20 \%$ of patients are alive 5 years after treatment $(71,72)$.

However, the actual standard treatment for localregionally advanced NSCLC which is not resectable was set up based on the results of the PACIFIC trial (NCT02125461) $(73,74)$. In this study, 713 patients with unresectable stage III NSCLC were randomly assigned at 1:2 to standard chemoradiation or chemoradiation followed by an anti-PD-L1 antibody, durvalumab (administered intravenously $10 \mathrm{mg}$ per kilogram, every 2 weeks for up 12 months). Primary endpoints were overall survival (OS) at 24 months and progression free survival (PFS), both of which resulted in a significant improvement in the durvalumab $\operatorname{arm}(66.3 \%$ OS and $17.2 \%$ PFS) vs. the placebo arm (55.6\% and $5.6 \%$, respectively). Key secondary endpoints shown improved results in the durvalumab groups $v s$. placebo; considering overall response rate $(30 \%$ vs. $17.8 \%)$, median duration of response (not reached at $27.4 v s .18 .4 \%$ ), time to death or distant metastasis (28.3 vs. 16.2), and time to second progression (28.3 vs. 17.1). Incidence of metastasis was lower in the durvalumab arm (22.5\% vs. $33.8 \%)$, and also patients receiving durvalumab had a lower incidence of brain metastasis ( $6.3 \%$ vs. $11.8 \%$ ). No any-grade all-causality adverse events (AEs) showed a significant difference between groups, and even though any-grade pneumonitis trended to be higher in the durvalumab group ( $33.9 \%$ vs. $24.8 \%$ ), grade $3 / 4$ pneumonitis rates were similar (3.6\% in the durvalumab arm vs. $3 \%$ in the placebo). The clinical benefit in the PACIFIC trial did not correlated with PD-L1 status, but it should be noted that PD-L1 testing was not mandatory, as no threshold was defined in inclusion criteria. PD-L1 status of $37 \%$ of patients was unknown, and safety outcomes were independent of PD-L1 too. Despite the U.S. Food and Drug Administration approved the use of durvalumab in local-regionally advanced NSCLC in February 2018, the European Medicines Agency limited the use of the ICI only to patients who were immunohistochemistry (IHC) positive.

Results from PACIFIC trial have focused new interest in therapies combining immunotherapy with other modalities of treatment. Several trials are actually recruiting patients when they have finished concurrent chemoradiation, and treating them with ICI. One to mention is the PACIFIC 6 (NCT03693300), which have changed the dose from 10 $\mathrm{mg} / \mathrm{kg}$ of durvalumab every 2 weeks administered in the original PACIFIC trial to $1,500 \mathrm{mg}$ every 4 weeks.

Other clinical trials are evaluating the effect of immunotherapy with concurrent chemoradiation followed by maintenance immunotherapy. The DETERRED trial (75) is currently ongoing, and it is divided in two parts, both of them consist of concurrent chemoradiation followed by 1 year of atezolizumab consolidation therapy, but differentiated in the administration of additional atezolizumab to concurrent chemoradiation in part 2. Its primary endpoints evaluate safety and feasibility, and results shown even higher grade 3 AEs in first group (40\% vs. $23 \%)$. There was no difference in OS between both groups (79\%), however PFS was relatively higher in part $2(50 \%$ in part 1 vs. $57 \%$ in part 2). However, evaluation of PD-L1 level showed no significant differences in cancer recurrence. In summary, the DETERRED trial demonstrated the safety of adding immunotherapy to concurrent chemoradiation.

Nowadays the real benefit of chemotherapy is being discussed, and it is theorized that the true benefit of chemoradiation belongs to radiosensitization, and that immunotherapy could reach the same clinical benefit more safely. Ongoing studies (NCT02221739 and NCT03391869) shows that radiotherapy combined with checkpoint inhibitors treatment is safe and tolerable. There is no strong evidence of an abscopal effect in immunotherapy, but the PACIFIC study improvements achieved in local-regional control and reduction in distant metastasis give clinical evidence that support this hypothesis.

The LUN 14-179 (NCT02343952) study is a phase II, single arm trial consisting of concurrent chemoradiation treatment followed by pembrolizumab in patients with unresectable stage III NSCLC. It showed efficacy and 
safety, and it achieved an OS $80.5 \%$ at 1 year and $68.7 \%$ at 2 years. Median PFS was 15,4 months; and 12, 18, and 24 -month PFS were $59.9 \%, 49.5 \%$ and $45,4 \%$ respectively (76).

In the phase II NICOLAS trial (NCT02434081), nivolumab is administered concurrently with radiotherapy followed by nivolumab consolidation therapy for up to 1 year. Eighty-two patients were recruited with median follow-up of 13.4 months. Safety analysis showed no unexpected AEs or increased toxicities, and most frequent were anemia, fatigue and pneumonitis. These results provide evidence that the addition of nivolumab to concurrent chemoradiotherapy is safe and tolerable. Following that, the 1-year PFS is still under evaluation and will be assessed in an expanded patient cohort (77).

The SPRINT trial (NCT03523702) is replacing concurrent chemotherapy with pembrolizumab in patients with high status of PD-L1 ( $>50 \%$ ), while patients under $50 \%$ are treated with concurrent chemoradiation. An additional trial (NCT03818776) is evaluating concurrent durvalumab with radiotherapy in patients with stage III NSCLC not eligible for concurrent chemoradiation therapy.

Even though the PACIFIC study represents a significant advance in the treatment of unresectable stage III NSCLC, there are multiple unknown factors such as the optimal timing of delivering of the immunotherapy, duration of immunotherapy administration, the most appropriate immunotherapeutic agent, the use of concomitant medications and possible autoimmune side effects that should be addressed with ongoing and future clinical trials, indexed in Table 3.

\section{Predictive biomarkers: PD-L1, TMB and emerging biomarkers}

The strikingly different behavior between the current standard of care and this new immunotherapy scheme, especially with chemoimmunotherapy, make the study of their molecular differences a key area for treatment improvement. However, due to the novelty of these therapies, the mechanism responsible for the differential response among patients to these therapies remains unknown.

TMB has recently emerged as a possible biomarker to predict NSCLC response. Primary targets of many tumor immune responses are neoantigen peptides derived from mutations, and high TMB usually correlates with higher response rates in carcinogen-driven cancers such as
NSCLC. In two independent cohorts of NSCLC patients treated with pembrolizumab, results shown an association between higher TMB and enhanced therapeutic efficacy, PFS and objective response (78), followed by significant associations between high TMB and ICI response in later NSCLC studies $(17,79-81)$. Furthermore, in the mentioned study a correlation between TMB and smoking status have been associated with better clinical benefit, as long as tumors from smokers have relatively high TMB (78). However, while the link between TMB and immunotherapy response is mostly solid, in some cases there are some patients with high TMB who are non-responders and vice versa.

On the other hand, it is supposed to be necessary certain expression of PD-L1 for anti-PD-1 therapy to have a real effect. Consequently, immunohistochemistry assays have been developed to define PD-L1 protein expression for clinical use. There are several clinical trials whose PD-L1 status correlates with response, such as KEYNOTE-001. In this study, the objective response rate among patients with PD-L1 $\geq 50 \%$ had a significant increase compared to all the patients (45\% vs. 19.4\%) (82). In NEOSTAR clinical trial, pre-treatment tumor PD-L1 levels was noteworthy higher in responders $v s$. non-responders ( $80 \%$ vs. $1 \%)$, and the median percentage of viable tumor was remarkable lower in tumors with PD-L $1>1 \%$ vs. PD-L $1 \leq 1 \%$ (20\% vs. $80 \%)$. Also in LCMC3 trial the percentage of patients with

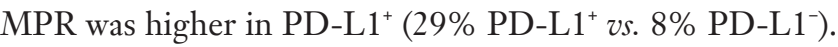
Additionally, 5 of 44 (11\%) with PD-L1 TPS $<50 \%$ and 7 of $20(35 \%)$ with PD-L1 TPS $>50 \%$ had MPR.

Despite this, determination of PD-L1 status remains an imperfect biomarker and not always is capable of predict an accurate immune response. Multiple studies in NSCLC tumors have detected no association between PDL1 status and response $(83,84)$. Potential reason for these contradictory results could be the temporal and spatial heterogeneous PD-L1 expression, the use of different detection assays, and non-standardized criteria and cut-offs for evaluating positivity. Recently, in the Keynote 189 phase III trial (85), PD-L1 status was predictive of response to pembrolizumab plus chemotherapy treatment at all levels of PD-L1. However, as the PD-L1 threshold was relaxed, the predictive effect of PD-L1 positivity notably decreased.

Regardless of the type of treatment, the neoadjuvant scenario is ideal as it allows us to study the pre-treatment immune status of a tumor (which would correspond to a tumor in the immune evasion phase) and, on the same individual, to study his post-treatment tumor, evaluating 


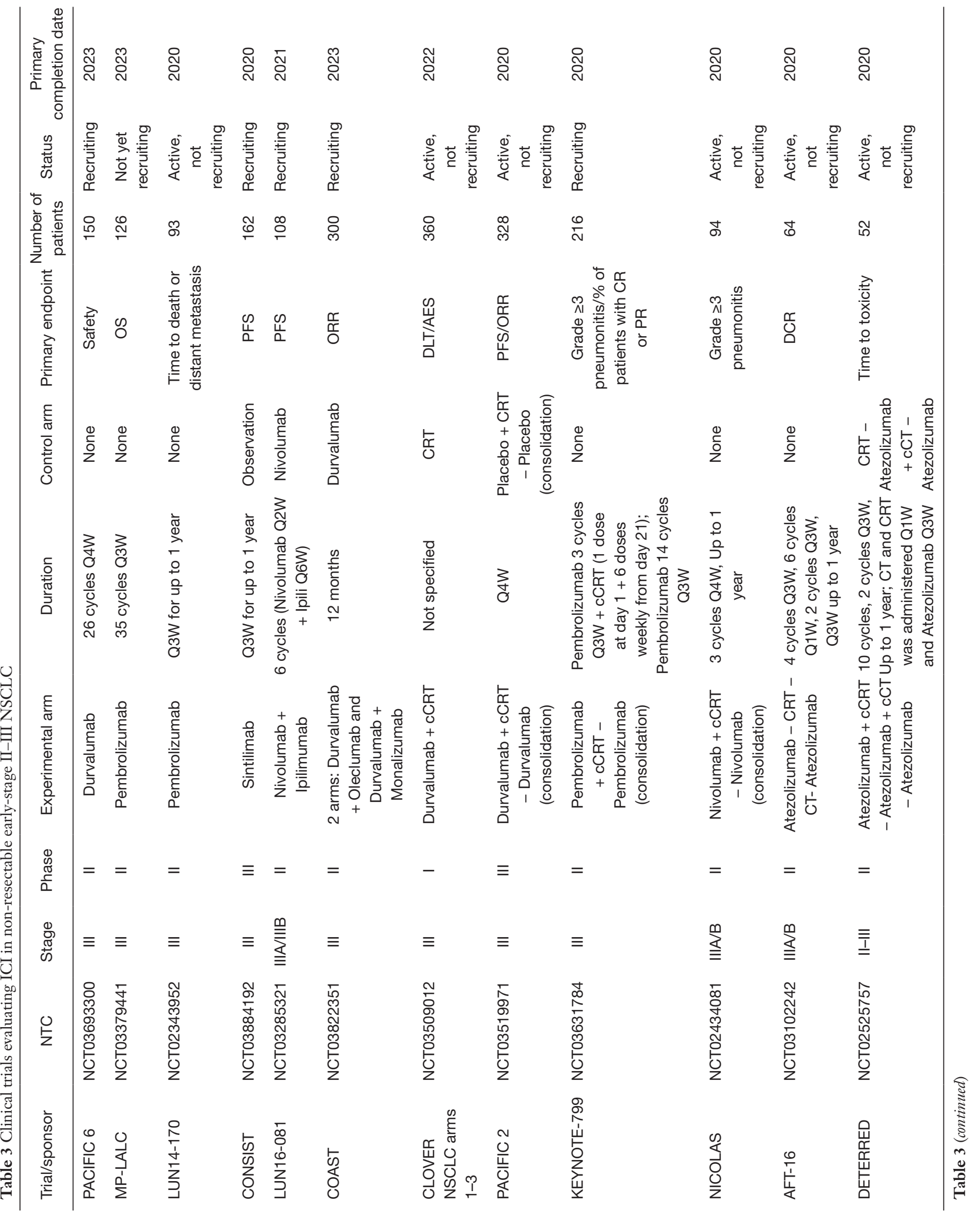




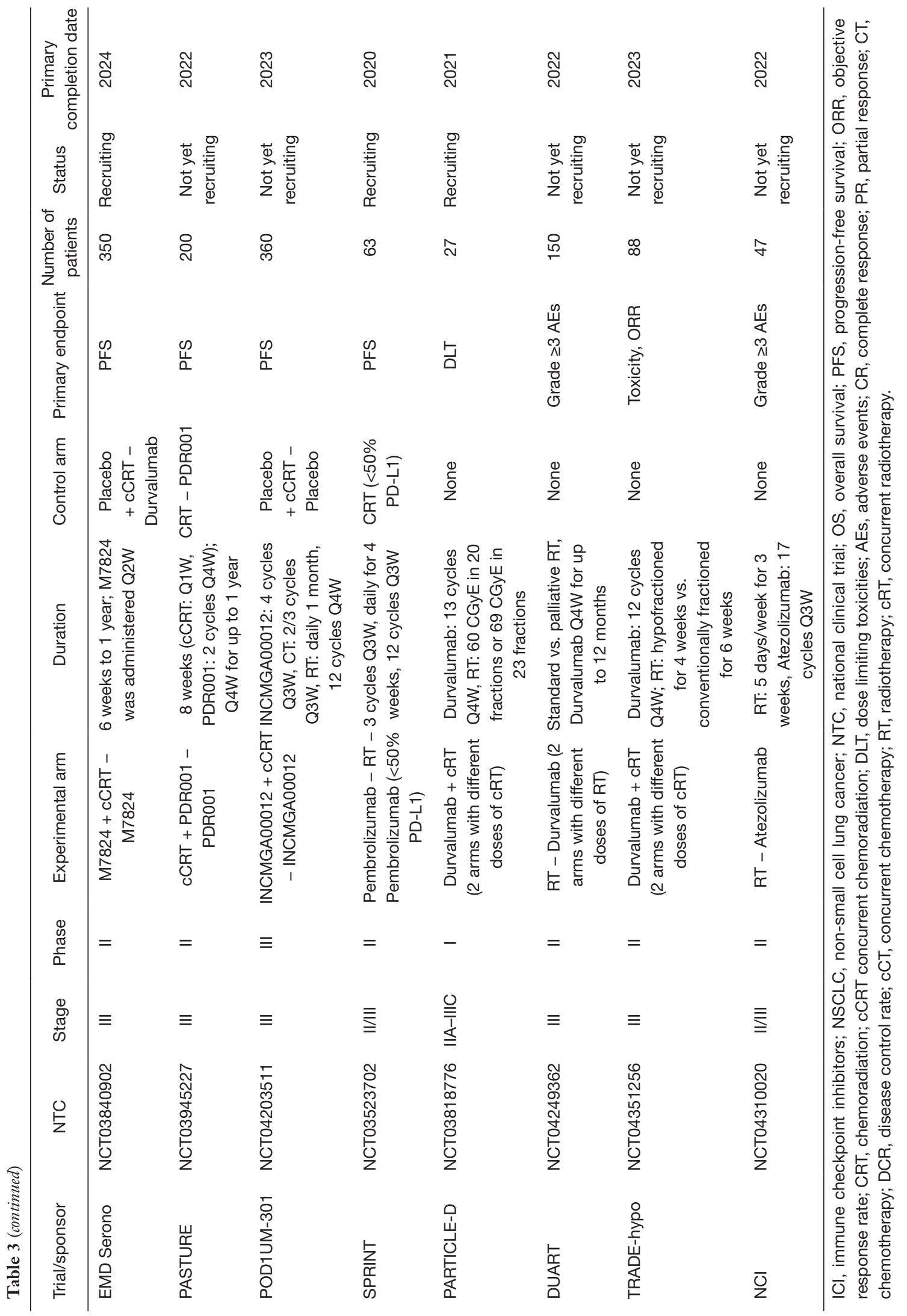


the changes in the tumor immune microenvironment and their relationship with the degree of treatment response and prognosis of the patient.

An interesting way to assess the status of the antitumor immune response is the deep sequencing of the TCR locus. During T cell maturation, their TCR locus suffer somatic rearrangements allowing $\mathrm{T}$ cells to recognize different antigens including tumor antigens. Cha et al., showed on melanoma and prostate patients treated with ipilimumab that massive sequencing on TCR locus from PBMCs allow them to correlate $\mathrm{T}$ cell repertoire with response to immunotherapy treatment (86). Later on, Akyüz et al., on another study with 18 patients treated with anti-PD-1 found a clear pattern of diversification of $\mathrm{T}$ cell clones correlated with the control of the disease (87). Recently, Forde et al. have described that the number of $\mathrm{T}$ cells clones shared in tumor and in peripheral blood increased after anti PD-1 treatment, in 8 of 9 patients with NSCLC (17). Therefore, the massive sequencing of the TCR locus is positioned as an interesting technique for the evaluation of the antitumor immune response.

\section{Study limitations}

Limitations of this overview include, but are not limited to, the intrinsic stage IIIA heterogeneous population, in which the definition of resectable $v$ s. unresectable is variable depending always on the decision of a multidisciplinary committee, hindering trials comparisons; addressing a topic with a large number of trials and fast development; discussing interim analysis results, that could change with final study conclusions; and focusing on immunotherapies based primarily on blocking PD-(L)1 or CTLA4, because of space constraints and current relevance.

\section{Conclusions}

Immunotherapy has revolutionized the treatment of advanced stages of lung cancer; becoming locally advanced stages its next challenge. As bring up in this review, numerous trials are currently evaluating the role of immunotherapy alone or in combination with other therapies, in both adjuvant and neoadjuvant setting.

Given the management complexity and intermediate prognosis of stage IIIA disease, circumstances such as the combination with other therapies, the treatment timing, and the patient selection through predictive markers, will be key to immunotherapy success in this scenario. Likewise, the increase in pathological response rates anticipated with immunotherapy opens the possibility to establish the MPR rate as a surrogate to neoadjuvant treatment. Preliminary results seem to favor neoadjuvant to adjuvant treatment, and through the NADIM trial, the chemoimmunotherapy combination seems to stand out.

In the next few years, the results of ongoing trials will answer whether immunotherapy can be implemented and to what extent is capable of transforming stage IIIA from a lethal condition to a curable disease.

\section{Acknowledgments}

Funding: Work in the authors' laboratories was supported by "Instituto de Salud Carlos III" (ISCIII) PI19/01652 grant, and European Union's Horizon 2020 research and innovation programme, CLARIFY 875160 grant, to M.P. A.C-B, received a Spanish Lung Cancer Group (SLCG) grant and is supported by a ISCIII "Sara Borrell" contract CD19/00170.

\section{Footnote}

Provenance and Peer Review: This article was commissioned by the Guest Editors (Fabrizio Minervini and Marco Scarci) for the series "Controversies in the Management of Stage IIIA Non-Small-Cell Lung Cancer" published in Current Challenges in Thoracic Surgery. The article has undergone external peer review.

Reporting Checklist: The authors have completed the Narrative Review reporting checklist. Available at https:// ccts.amegroups.com/article/view/10.21037/ccts-20-82/rc

Conflicts of Interest: All authors have completed the ICMJE uniform disclosure form (available at https://ccts. amegroups.com/article/view/10.21037/ccts-20-82/coif). The series "Controversies in the Management of Stage IIIA Non-Small-Cell Lung Cancer" was commissioned by the editorial office without any funding or sponsorship. VC reports personal fees from Roche, BMS, MSD, AstraZeneca, Boehringer, Novartis and Takeda, outside the submitted work. MP reports grants and personal fees from BMS, personal fees from Astra Zeneca, Roche, MSD and Takeda, outside the submitted work. The authors have no other conflicts of interest to declare.

Ethical Statement: The authors are accountable for all 
aspects of the work in ensuring that questions related to the accuracy or integrity of any part of the work are appropriately investigated and resolved.

Open Access Statement: This is an Open Access article distributed in accordance with the Creative Commons Attribution-NonCommercial-NoDerivs 4.0 International License (CC BY-NC-ND 4.0), which permits the noncommercial replication and distribution of the article with the strict proviso that no changes or edits are made and the original work is properly cited (including links to both the formal publication through the relevant DOI and the license). See: https://creativecommons.org/licenses/by-nc-nd/4.0/.

\section{References}

1. Siegel RL, Miller KD, Jemal A. Cancer statistics, 2020. CA Cancer J Clin 2020;70:7-30.

2. Goldstraw P, Chansky K, Crowley J, et al. The IASLC lung cancer staging project: Proposals for revision of the TNM stage groupings in the forthcoming (eighth) edition of the TNM Classification for lung cancer. J Thorac Oncol 2016;11:39-51.

3. Rami-Porta R, Call S, Dooms C, et al. Lung cancer staging: A concise update. Eur Respir J 2018;51:1800190.

4. Majem M, Hernández-Hernández J, Hernando-Trancho F, et al. Multidisciplinary consensus statement on the clinical management of patients with stage III non-small cell lung cancer. Clin Transl Oncol 2020;22:21-36.

5. Ramnath N, Dilling TJ, Harris LJ, et al. Treatment of stage III non-small cell lung cancer: Diagnosis and management of lung cancer, 3rd ed: American college of chest physicians evidence-based clinical practice guidelines. Chest 2013;143:e314S-e340S.

6. Sharma P, Allison JP. Immune checkpoint targeting in cancer therapy: Toward combination strategies with curative potential. Cell 2015;161:205-214.

7. Iafolla MAJ, Juergens RA. Update on Programmed Death-1 and Programmed Death-Ligand 1 Inhibition in the Treatment of Advanced or Metastatic Non-Small Cell Lung Cancer. Front Oncol 2017;7:67.

8. O'Donnell JS, Teng MWL, Smyth MJ. 2018. Cancer immunoediting and resistance to $\mathrm{T}$ cell-based immunotherapy. Nat Rev Clin Oncol 2019;16:151-67.

9. Binnewies M, Roberts EW, Kersten K, et al. Understanding the tumor immune microenvironment (TIME) for effective therapy. Nat Med 2018;24:541-550.

10. Vinay DS, Ryan EP, Pawelec G, et al. Immune evasion in cancer: Mechanistic basis and therapeutic strategies. Semin Cancer Biol 2015;35:S185-98.

11. Mittal D, Gubin MM, Schreiber RD, et al. New insights into cancer immunoediting and its three component phases-elimination, equilibrium and escape. Curr Opin Immunol 2014;27:16-25.

12. Takada K, Okamoto T, Shoji F, et al. Clinical significance of PD-L1 protein expression in surgically resected primary lung adenocarcinoma. J Thorac Oncol 2016;11:1879-90.

13. Zhang M, Li G, Wang Y, et al. PD-L1 expression in lung cancer and its correlation with driver mutations: a metaanalysis. Sci Rep 2017;7:10255.

14. Yost KE, Satpathy AT, Wells DK, et al. Clonal replacement of tumor-specific T cells following PD-1 blockade. Nat Med 2019;25:1251-9.

15. Ribas A, Shin DS, Zaretsky J, et al. PD-1 blockade expands intratumoral memory T cells. Cancer Immunol Res 2016;4:194-203.

16. Ribas A, Wolchok JD. Cancer immunotherapy using checkpoint blockade. Science 2018;359:1350-5.

17. Forde PM, Chaft JE, Smith KN, et al. Neoadjuvant PD-1 Blockade in Resectable Lung Cancer. N Engl J Med 2018;378:1976-86.

18. Topalian SL, Taube JM, Pardoll DM. Neoadjuvant checkpoint blockade for cancer immunotherapy. Science 2020;367:eaax0182.

19. Bakos O, Lawson C, Rouleau S, et al. Combining surgery and immunotherapy: Turning an immunosuppressive effect into a therapeutic opportunity. J Immunother Cancer 2018;6:86.

20. Kim JM, Chen DS. Immune-escape to PD-L1/PD-1 blockade: 7 steps to success (or failure). Ann Oncol 2016;27:1492-504.

21. Festino L, Botti G, Lorigan P, et al. Cancer Treatment with Anti-PD-1/PD-L1 Agents: Is PD-L1 Expression a Biomarker for Patient Selection? Drugs 2016;76:925-45.

22. Pitroda SP, Chmura SJ, Weichselbaum RR. Integration of radiotherapy and immunotherapy for treatment of oligometastases. Lancet Oncol 2019;20:e434-42.

23. Provencio-Pulla M, Nadal-Alforja E, Cobo M, et al. Neoadjuvant chemo/immunotherapy for the treatment of stages IIIA resectable non-small cell lung cancer (NSCLC): A phase II multicenter exploratory study-NADIM studySLCG. J Clin Oncol 2018;36:8521.

24. Shu CA, Grigg C, Chiuzan C, et al. Neoadjuvant atezolizumab + chemotherapy in resectable non-small cell lung cancer (NSCLC). J Clin Oncol 2018;36:8532.

25. Bracci L, Schiavoni G, Sistigu A, et al. Immune-based 
mechanisms of cytotoxic chemotherapy: implications for the design of novel and rationale-based combined treatments against cancer. Cell Death Differ 2014;21:15-25.

26. Parra ER, Villalobos P, Behrens C, et al. Effect of neoadjuvant chemotherapy on the immune microenvironment in non-small cell lung carcinomas as determined by multiplex immunofluorescence and image analysis approaches. J Immunother Cancer 2018;6:48.

27. Zhang L, Dermawan K, Jin M, et al. Differential impairment of regulatory $\mathrm{T}$ cells rather than effector $\mathrm{T}$ cells by paclitaxel-based chemotherapy. Clin Immunol 2008;129:219-229.

28. Zhu Y, Liu N, Xiong SD, et al. CD4+Foxp3+ Regulatory T-cell Impairment by Paclitaxel is Independent of Toll-like Receptor 4. Scand J Immunol 2011;73:301-8.

29. Sim SH, Ahn YO, Yoon J, et al. Influence of chemotherapy on nitric oxide synthase, indole-amine-2,3-dioxygenase and CD124 expression in granulocytes and monocytes of non-small cell lung cancer. Cancer Sci 2012;103:155-60.

30. Douillard JY, Rosell R, De Lena M, et al. Adjuvant vinorelbine plus cisplatin versus observation in patients with completely resected stage IB-IIIA non-small-cell lung cancer (Adjuvant Navelbine International Trialist Association [ANITA]): a randomised controlled trial. Lancet Oncol 2006;7:719-27.

31. Pignon JPP, Tribodet H, Scagliotti GV, et al. Lung adjuvant cisplatin evaluation: A pooled analysis by the LACE collaborative group. J Clin Oncol 2008;26:3552-9.

32. Burdett S, Rydzewska L, Tierney J, et al. Postoperative radiotherapy for non-small cell lung cancer. Cochrane Database Syst Rev 2016;10:CD002142.

33. Robinson CG, Patel AP, Bradley JD, et al. Postoperative radiotherapy for pathologic $\mathrm{N} 2$ non-small-cell lung cancer treated with adjuvant chemotherapy: A review of the national cancer data base. J Clin Oncol 2015;33:870-6.

34. Postmus PE, Kerr KM, Oudkerk M, et al. Early and locally advanced non-small-cell lung cancer (NSCLC): ESMO Clinical Practice Guidelines for diagnosis, treatment and follow-up. Ann Oncol 2017;28:iv1-iv21.

35. Moujaess E, Haddad FG, Eid R, et al. The emerging use of immune checkpoint blockade in the adjuvant setting for solid tumors: a review. Immunotherapy 2019;11:1409-22.

36. Eggermont AMM, Chiarion-Sileni V, Grob JJ, et al. Prolonged survival in stage III melanoma with ipilimumab adjuvant therapy. N Engl J Med 2016;375:1845-55.

37. Weber J, Mandala M, Del Vecchio M, et al. Adjuvant nivolumab versus ipilimumab in resected stage III or IV melanoma. N Engl J Med 2017;377:1824-35.

38. Eggermont AMM, Blank CU, Mandala M, et al. Adjuvant pembrolizumab versus placebo in resected stage III melanoma. N Engl J Med 2018;378:1789-801.

39. Chaft JE, Dahlberg SE, Khullar O V, et al. EA5142 adjuvant nivolumab in resected lung cancers (ANVIL). J Clin Oncol 2018;36:TPS8581.

40. O'Brien MER, Hasan B, Dafni U, et al. EORTC-ETOP randomized, phase 3 trial with anti-PD-1 monoclonal antibody pembrolizumab versus placebo for patients with early stage non-small cell lung cancer (NSCLC) after resection and standard adjuvant chemotherapy: PEARLS (NCT02504372). J Clin Oncol 2016;34:TPS8571.

41. Felip E, Wakelee H, Vallieres E, et al. P2.04-004 IMpower010: A Phase III Study of Atezolizumab vs. Best Supportive Care Following Adjuvant Chemotherapy in Completely Resected NSCLC. J Thorac Oncol 2017;12:S2398-9.

42. Burdett S, Stewart LA, Rydzewska L. A systematic review and meta-analysis of the literature: Chemotherapy and surgery versus surgery alone in non-small cell lung cancer. J Thorac Oncol 2006;1:611-21.

43. Nagai K, Tsuchiya R, Mori T, et al. A randomized trial comparing induction chemotherapy followed by surgery with surgery alone for patients with stage IIIA N2 nonsmall cell lung cancer (JCOG 9209). J Thorac Cardiovasc Surg 2003;125:254-60.

44. Rosell R, Gómez-Codina J, Camps C, et al. Preresectional chemotherapy in stage IIIA non-small-cell lung cancer: A 7-year assessment of a randomized controlled trial. Lung Cancer 1999;26:7-14.

45. Rosell R, Gomez-Codina J, Camps C, et al. A Randomized Trial Comparing Preoperative Chemotherapy Plus Surgery with Surgery Alone in Patients with Non-SmallCell Lung Cancer. N Engl J Med 1994;330:153-8.

46. Roth JA, Fossella F, Komaki R, et al. A Randomized Trial Comparing Perioperative Chemotherapy and Surgery With Surgery Alone in Resectable Stage IIIA Non-SmallCell Lung Cancer. J Natl Cancer Inst 1994;86:673-80.

47. NSCLC Meta-analysis Collaborative Group. Preoperative chemotherapy for non-small-cell lung cancer: A systematic review and meta-analysis of individual participant data. Lancet 2014;383:1561-71.

48. Kirn DH, Lynch TJ, Mentzer SJ, et al. Multimodality therapy of patients with stage IIIA, N2 non-small-cell lung cancer: Impact of preoperative chemotherapy on resectability and downstaging. J Thorac Cardiovasc Surg 1993;106:696-702. 
49. Sugarbaker DJ, Herndon J, Kohman LJ, et al. Results of cancer and leukemia group B protocol 8935. A multiinstitutional phase II trimodality trial for stage IIIA (N2) non-small-cell lung cancer. Cancer and Leukemia Group B Thoracic Surgery Group. J Thorac Cardiovasc Surg 1995;109:473-83; discussion 483-5.

50. Choi YS, Shim YM, Kim J, et al. Recurrence-free survival and prognostic factors in resected pN2 non-small cell lung cancer. Eur J Cardiothorac Surg 2002;22:695-700.

51. Hellmann MD, Chaft JE, William WN, et al. Pathological response after neoadjuvant chemotherapy in resectable non-small-cell lung cancers: proposal for the use of major pathological response as a surrogate endpoint. Lancet Oncol 2014;15:e42-50.

52. Pataer A, Kalhor N, Correa AM, et al. Histopathologic response criteria predict survival of patients with resected lung cancer after neoadjuvant chemotherapy. J Thorac Oncol 2012;7:825-32.

53. Junker K, Langner K, Klinke F, et al. Grading of tumor regression in non-small cell lung cancer: Morphology and prognosis. Chest 2001;120:1584-91.

54. Chemotherapy in Non-Small Cell Lung Cancer: A MetaAnalysis Using Updated Data on Individual Patients From 52 Randomised Clinical Trials. Non-small Cell Lung Cancer Collaborative Group. BMJ 1995;311:899-909.

55. Cottrell TR, Thompson ED, Forde PM, et al. Pathologic features of response to neoadjuvant anti-PD-1 in resected non-small-cell lung carcinoma: A proposal for quantitative immune-related pathologic response criteria (irPRC). Ann Oncol 2018;29:1853-60.

56. Travis WD, Dacic S, Wistuba I, et al. IASLC Multidisciplinary Recommendations for Pathologic Assessment of Lung Cancer Resection Specimens Following Neoadjuvant Therapy. Available online: https:// www.jto.org/article/S1556-0864(20)30026-5/fulltext

57. Gerwing M, Herrmann K, Helfen A, et al. The beginning of the end for conventional RECIST - novel therapies require novel imaging approaches. Nat Rev Clin Oncol 2019;16:442-58.

58. Provencio M, Nadal E, Insa A, et al. OA13.05 NADIM Study: Updated Clinical Research and Outcomes. J Thorac Oncol 2019;14:S241.

59. Reuss JE, Smith KN, Anagnostou V, et al. Neoadjuvant nivolumab in resectable non-small cell lung cancer: Extended follow-up and molecular markers of response. J Clin Oncol 2019;37:8524.

60. Rusch VW, Chaft JE, Johnson B, et al. Neoadjuvant atezolizumab in resectable non-small cell lung cancer
(NSCLC): Initial results from a multicenter study (LCMC3). J Clin Oncol 2018;36:8541.

61. Kwiatkowski DJ, Rusch VW, Chaft JE, et al. Neoadjuvant atezolizumab in resectable non-small cell lung cancer (NSCLC): Interim analysis and biomarker data from a multicenter study (LCMC3). J Clin Oncol 2019;37:8503.

62. Cascone T, William WN, Weissferdt A, et al. Neoadjuvant nivolumab $(\mathrm{N})$ or nivolumab plus ipilimumab (NI) for resectable non-small cell lung cancer (NSCLC). Ann Oncol 2018;29:viii738.

63. Cascone T, William WN, Weissferdt A, et al. Neoadjuvant nivolumab $(\mathrm{N})$ or nivolumab plus ipilimumab $(\mathrm{NI})$ for resectable non-small cell lung cancer (NSCLC): Clinical and correlative results from the NEOSTAR study. J Clin Oncol 2019;37:8504.

64. Shu CA, Gainor JF, Awad MM, et al. Articles Neoadjuvant atezolizumab and chemotherapy in patients with resectable non-small-cell lung cancer: an open-label, multicentre, single-arm, phase 2 trial. Lancet Oncol 2020;21:786-95.

65. Fernando HC, Yang J, Ferraro GL, et al. Randomized, double-blind phase 3 study evaluating neoadjuvant platinum-based chemotherapy with perioperative pembrolizumab or placebo in resectable stage IIB or IIIA NSCLC: KEYNOTE-671. J Clin Oncol 2018;36:TPS8583.

66. Felip E, Brahmer J, Broderick S, et al. P2.16-03 CheckMate 816: A Phase 3 Trial of Neoadjuvant Nivolumab Plus Ipilimumab or Chemotherapy vs. Chemotherapy in Early-Stage NSCLC. J Thorac Oncol 2018;13:S831-2.

67. Rizvi N, Gandara D, Solomon B, et al. P2.17-27 IMpower030: Phase III Study Evaluating Neoadjuvant Treatment of Resectable Stage II-IIIB NSCLC with Atezolizumab + Chemotherapy. J Thorac Oncol 2018;13:S863.

68. Schaake-Koning C, Van den Bogaert W, Dalesio O, et al. Effects of concomitant cisplatin and radiotherapy on inoperable non-small-cell lung cancer. N Engl J Med 1992;326:524-30.

69. Jeremic B, Shibamoto Y, Acimovic L, et al. Hyperfractionated radiation therapy with or without concurrent low-dose daily carboplatin/etoposide for stage III non-small-cell lung cancer: a randomized study. J Clin Oncol 1996;14:1065-70.

70. Curran WJ, Paulus R, Langer CJ, et al. Sequential vs. concurrent chemoradiation for stage iii non-small cell lung cancer: Randomized phase III trial RTOG 9410. J Natl Cancer Inst 2011;103:1452-60. 
71. Aupérin A, Le Péchoux C, Rolland E, et al. Meta-analysis of concomitant versus sequential radiochemotherapy in locally advanced non - small-cell lung cancer. J Clin Oncol 2010;28:2181-90.

72. Ahn JS, Ahn YC, Kim JH, et al. Multinational randomized phase III trial with or without consolidation chemotherapy using docetaxel and cisplatin after concurrent chemoradiation in inoperable stage III non-small-cell lung cancer: KCSG-LU05-04. J Clin Oncol 2015;33:2660-6.

73. Antonia SJ, Villegas A, Daniel D, et al. Overall survival with durvalumab after chemoradiotherapy in stage III NSCLC. N Engl J Med 2018;379:2342-50.

74. Antonia SJ, Villegas A, Daniel D, et al. Durvalumab after chemoradiotherapy in stage III non-small-cell lung cancer. N Engl J Med 2017;377:1919-29.

75. Lin SH, Lin Y, Mok I, et al. Phase II trial combining atezolizumab concurrently with chemoradiation therapy in locally advanced non-small cell lung cancer. J Clin Oncol 2019;37:8512.

76. Durm GA, Althouse SK, Sadiq AA, et al. Phase II trial of concurrent chemoradiation with consolidation pembrolizumab in patients with unresectable stage III nonsmall cell lung cancer: Hoosier Cancer Research Network LUN 14-179. J Clin Oncol 2018;36:8500.

77. Peters S, Felip E, Dafni U, et al. Safety evaluation of nivolumab added concurrently to radiotherapy in a standard first line chemo-radiotherapy regimen in stage III non-small cell lung cancer-The ETOP NICOLAS trial. Lung Cancer 2019;133:83-7.

78. Rizvi NA, Hellmann MD, Snyder A, et al. Mutational landscape determines sensitivity to PD-1 blockade in nonsmall cell lung cancer. Science 2015;348:124-8.

79. Hellmann MD, Nathanson T, Rizvi H, et al. Genomic

doi: $10.21037 /$ ccts-20-82

Cite this article as: Calvo V, Sierra-Rodero B, Cruz-Bermúdez A, Provencio M. Role of immunotherapy in stage IIIA nonsmall cell lung cancer: a narrative review. Curr Chall Thorac Surg 2021;3:38.
Features of Response to Combination Immunotherapy in Patients with Advanced Non-Small-Cell Lung Cancer. Cancer Cell 2018;33:843-52.e4.

80. Hellmann MD, Ciuleanu TE, Pluzanski A, et al. Nivolumab plus ipilimumab in lung cancer with a high tumor mutational burden. N Engl J Med 2018;378:2093-104.

81. Jermann P, Alborelli I, Leonards K, et al. Tumor mutational burden assessed by a targeted NGS assay predicts clinical benefit from immune checkpoint inhibitors in non-small cell lung cancer. Ann Oncol 2019;30:ii59.

82. Garon EB, Rizvi NA, Hui R, et al. Pembrolizumab for the treatment of non-small-cell lung cancer. N Engl J Med 2015;372:2018-28.

83. Carbone DP, Reck M, Paz-Ares L, et al. First-line nivolumab in stage IV or recurrent non-small-cell lung cancer. N Engl J Med 2017;376:2415-26.

84. Brahmer J, Reckamp KL, Baas P, et al. Nivolumab versus docetaxel in advanced squamous-cell non-small-cell lung cancer. N Engl J Med 2015;373:123-35.

85. Gandhi L, Rodríguez-Abreu D, Gadgeel S, et al. Pembrolizumab plus Chemotherapy in Metastatic Non-Small-Cell Lung Cancer. N Engl J Med 2018;378:2078-92.

86. Cha E, Klinger M, Hou Y, et al. Improved Survival with T Cell Clonotype Stability After Anti - CTLA-4 Treatment in Cancer Patients. Sci Transl Med 2014;6:238ra70.

87. Akyüz N, Brandt A, Stein A, et al. T-cell diversification reflects antigen selection in the blood of patients on immune checkpoint inhibition and may be exploited as liquid biopsy biomarker. Int J Cancer 2017;140:2535-44. 